\title{
Three Theorems, with Computer-Aided Proofs, on Three-Dimensional Faces and Quotients of Polytopes
}

\author{
G. Meisinger, ${ }^{1}$ P. Kleinschmidt, ${ }^{2}$ and G. Kalai ${ }^{3}$ \\ ${ }^{1}$ SERKEM Gesellschaft für IT-Services und Consulting, \\ Watzmannsdorfer Ring 27, 94136 Thyrnau, Germany \\ guenter.meisinger@serkem.de \\ ${ }^{2}$ Lehrstuhl für Wirtschaftsinformatik, Universität Passau, \\ Innstrasse 29, 94032 Passau, Germany \\ kleinsch@winf.uni-passau.de \\ ${ }^{3}$ Institute of Mathematics, Hebrew University, \\ Jerusalem, Israel \\ kalai@math.huji.ac.il
}

\begin{abstract}
We prove that there is a finite list of 3-polytopes so that every rational $d$ polytope, $d \geq 9$, contains a three-dimensional face in the list. A similar result where "faces" are replaced by "quotients" is proved already for (general) 5-polytopes. We also prove that every $d$-polytope, $d \geq 9$, contains a three-dimensional quotient which is a simplex.
\end{abstract}

\section{Introduction}

Theorem 1. There is a finite list of three-dimensional polytopes such that every rational 9-polytope contains a three-dimensional face in the list.

Here, a rational polytope is a polytope whose vertices have rational coordinates. Duals of neighborly 4-polytopes show that the dimension 9 cannot be reduced to 4 .

Theorem 2. Every nine-dimensional polytope has the three-dimensional simplex as a quotient.

The 24-cell shows that, here too, the dimension 9 cannot be reduced to 4 .

The analogous situation for two-dimensional faces and quotients is classic. It follows easily from Euler's theorem that every 3-polytope (and hence every higher-dimensional 
polytope) has a two-dimensional face which is a triangle, quadrangle, or a pentagon. In fact, this result (in a dual form) can be traced back to the writings of Descartes on polyhedra, see [4]. It also follows easily from Euler's theorem that every 3-polytope or its dual contains a triangular face and thus every $d$-polytope, $d \geq 3$, contains a triangle as a quotient.

Theorems 1 and 2 are special cases of some far-reaching conjectures, see [11]: Define for every $k>1$ four functions $d_{1}=d_{1}(k), d_{2}=d_{2}(k), d_{3}=d_{3}(k)$, and $d_{4}=d_{4}(k)$ to be the smallest integers so that:

- There is a finite list of $k$-polytopes so that every $d$-polytope, $d \geq d_{1}(k)$, has a $k$-dimensional quotient in the list.

- There is a finite list of $k$-polytopes so that every $d$-polytope, $d \geq d_{2}(k)$, contains a $k$-face in the list.

- Every $d$-polytope, $d \geq d_{3}(k)$, has a simplex as a $k$-dimensional quotient.

- Every $d$-polytope, $d \geq d_{4}(k)$, contains a $k$-face which is combinatorially isomorphic to a simplex or to a cube.

It is conjectured that all these four functions are finite. This will be easiest to prove for $d_{1}$ and hardest for $d_{4}$. Clearly, $d_{1}(k) \leq d_{3}(k) \leq d_{4}(k+1)$ and $d_{1}(k) \leq d_{2}(k) \leq d_{4}(k)$. The following theorem gives that $d_{1}(3) \leq 5$. We do not know if the correct value for $d_{1}(3)$ is 4 or 5 . We can prove also that $d_{1}(4) \leq 7 . d_{4}(2)=5[11]$.

Theorem 3. Every 5-polytope contains a three-dimensional quotient with at most eight vertices.

The proofs of Theorems 1-3 as a consequence of (rather deep) known inequalities for flag numbers of polytopes were achieved by a computerized program FLAGTOOL, see [12] and [14]. A comprehensive description of FLAGTOOL and theorems proved by FLAGTOOL has appeared in [14].

This work is closely related to various ideas and results by Grünbaum. The proof uses the lower bound theorem for polytopes and some of its far-reaching generalizations. Some of these generalizations are based on the rigidity theory for polytopes, a topic of "lost mathematics" Grünbaum helped to revive [8]. The type of reasoning (convolutions) used here also has roots in some early papers of Grünbaum. However, more than that, we feel these proofs touch on some fundamental issues concerning mathematical interest, elegance in mathematics, and the use of computers raised by Grünbaum in various places, see [5]-[7].

\section{Face Numbers, Flag Numbers, $g$-Numbers, and Convolutions}

For a $d$-polytope $P$ the number of $k$-faces is denoted by $f_{k}(P)$. (We also use the notation $f_{k}^{d}$ unless the value of $d$ is clear from the context.) The vector $\left(f_{0}(P), f_{1}(P), \ldots f_{d-1}(P)\right)$ is called the $f$-vector of $P$. For a subset $S=\left\{i_{1}, \ldots, i_{k}\right\} \subset\{0,1, \ldots, d-1\}$ the flag number $f_{S}(P)$ is the number of chains $F_{1} \subset F_{2} \subset \cdots \subset F_{k}$ of faces of $P$ such that $\operatorname{dim} F_{j}=i_{j}$. (Again, we also use the notation $f_{S}^{d}$.) A remarkable theorem of Bayer and Billera asserts that the affine dimension of the space of flag numbers of $d$-polytopes is 
$c_{d}-1$, where $c_{d}$ is the $d$ th Fibonacci number. Bayer and Billera showed that every flag number $f_{S}^{d}$ can be expressed as a linear combination of special flag numbers $f_{T}^{d}$, where $T \subset\{0,1, \ldots, d-2\}$ and $T$ contains no two consecutive integers. Their argument relies only on Euler's formula (for arbitrary dimension) and it therefore applies not only for polytopes but for arbitrary Eulerian posets, see [17].

Certain linear combinations of face numbers of simplicial polytopes called $h$-numbers and $g$-numbers play a crucial role in the combinatorial theory of simplicial polytopes, see [13] and [16]. Intersection homology theory has led to deep and mysterious extensions of $h$ - and $g$-numbers from simplicial polytopes to general polytopes. The definition (which can also be found in [15]) goes as follows. For a polytope $P$ denote by $P_{k}$ the set of $k$-faces of $P$.

Define by induction two polynomials

$$
h_{P}(x)=\sum_{k=0}^{d} h_{k}^{d} x^{d-k}, \quad g_{P}(x)=\sum_{k=0}^{[d / 2]} g_{k}^{d} x^{d-k},
$$

by the rules: (a) $g_{k}^{d}=h_{k}^{d}-h_{k-1}^{d}$, (b) if $P$ is the empty polytope or a 0 -polytope $P$, $h_{P}=g_{P}=1$, and

$$
h_{P}(x)=\sum_{k=0}^{d}(x-1)^{d-k} \sum\left\{g_{F}(x): x \in P_{k}\right\}
$$

(If the value of $d$ is clear from the context the superscripts of $h_{k}^{d}$ and $g_{k}^{d}$ are omitted.)

Thus $g_{1}^{d}(P)=f_{0}(P)-d-1$ and

$$
g_{2}^{d}(P)=f_{1}(P)+\sum\left\{f_{0}(F)-3: F \in P_{2}\right\}-d f_{0}(P)+\left(\begin{array}{c}
d+1 \\
2
\end{array}\right) .
$$

The value of $g_{2}^{d}$ for general $d$-polytopes has a rigidity theoretic meaning [9] and is nonnegative for every polytope. This extends the famous lower bound theorem of Barnette [1]. The nonnegativity of $g_{2}^{d}$ is still open for more general objects like polyhedral $(d-1)$-spheres and manifolds. It follows from intersection homology theory for toric varieties that the $g_{k}^{d}$ are nonnegative for every rational $d$-polytope. This is still open in the nonrational case. For a $d$-polytope $P$ we denote $\bar{g}_{k}^{d}(P)=g_{k}^{d}\left(P^{*}\right)$, where $P$ is the dual polytope to $P$.

Let $m^{d}, m^{e}$ be linear combinations of flag numbers of $d$-and $e$-polytopes, respectively. For a polytope $P$ of dimension $d+e+1$ define the convolution of $m^{d}$ and $m^{e}$ by $m^{d} * m^{e}(P)=\sum\left\{m^{d}(F) \cdot m^{e}(P / F): F\right.$ a $d$-face of $\left.P\right\}$.

The following lemma [10] is immediate:

\section{Lemma 4.}

(1) $m^{d} * m^{e}(P)$ is a linear combination of flag numbers of $(d+e+1)$-polytopes.

(2) If $m^{d}(P)=0$ for every d-polytope $P$ or $m^{e}(Q)=0$ for every e-polytope $Q$, then $m^{d} * m^{e}(R)=0$ for every $(d+e+1)$-polytope $R$.

(3) If $m^{d}(P) \geq 0$ for every d-polytope $P$ and $m^{e}(Q) \geq 0$ for every e-polytope $Q$, then $m^{d} * m^{e}(R) \geq 0$ for every $(d+e+1)$-polytope $R$. 


\section{FLAGTOOL}

FLAGTOOL [14], [12] is a computer program that

- computes all (known) linear relations between the flag numbers of general $d$ polytopes for small dimensions, $3 \leq d \leq 10$,

- extracts and automatically proves new results from those relations.

Since every flag number can be expressed as an affine combination of special flag numbers, all inequalities generated by the program will be expressed in terms of special flag numbers. See Fig. 1 for the general scheme supporting theorem proving with FLAGTOOL.

Theorem 1 is a consequence of the following stronger statement.

Theorem 5. Every rational d-polytope $(d \geq 9)$ has a 3-face with less than 78 vertices or 78 facets.

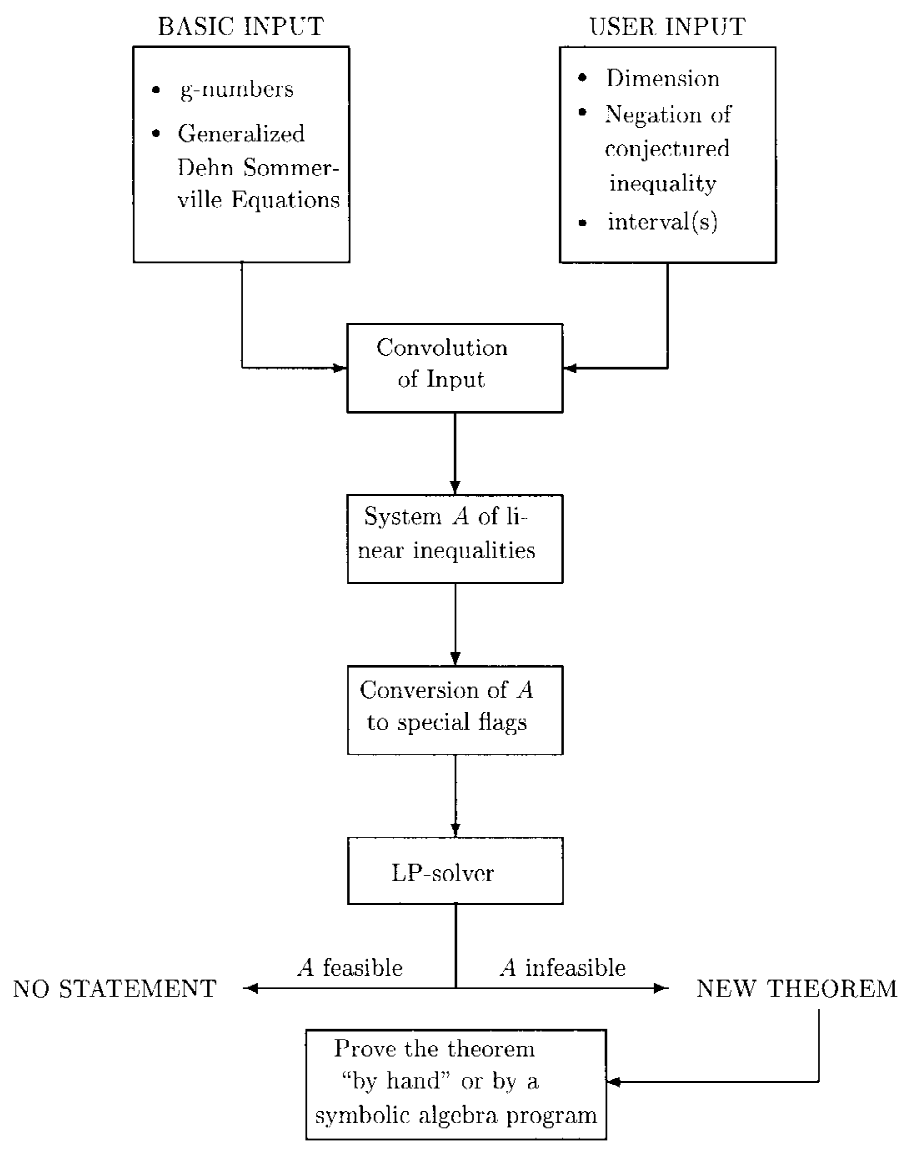

Fig. 1. Automated theorem proving by FLAGTOOL. 
Proof. Assume that there exists a 9-polytope in which every 3-face has at least 78 vertices and at least 78 facets. This assumption can be expressed by the inequalities $f_{0}^{3}-78 \geq 0$ and $f_{2}^{3}-78 \geq 0$. The following system of 53 linear 9 -forms obtained by convolutions of the $g$-numbers and of these two added inequalities (in the bottom interval $[-1,3])$ has no nonnegative feasible solution and therefore Theorem 1 is proved.

$$
\begin{aligned}
g_{0}^{1} * g_{1}^{2} * g_{0}^{0} * g_{1}^{2} * g_{0}^{0}= & 6 f_{0246}-18 f_{025}+6 f_{135}+36 f_{15}-18 f_{146}-3 f_{0257} \\
& +f_{1357}+6 f_{157}, \\
g_{1}^{2} * g_{1}^{2} * g_{1}^{2} * g_{0}^{0}= & -54 f_{25}-9 f_{257}-54 f_{36}+27 f_{036}+54 f_{26}-18 f_{035} \\
& +36 f_{35}+9 f_{246}-3 f_{0357}+6 f_{357}+18 f_{025}+3 f_{0257} \\
& -9 f_{136}-18 f_{026}+6 f_{135}-3 f_{0246}+f_{1357}, \\
g_{0}^{0} * g_{2}^{4} * g_{1}^{2} * g_{0}^{0}= & -60 f_{05}-10 f_{057}-60 f_{16}-30 f_{036}+60 f_{06}+48 f_{15} \\
& +8 f_{157}-12 f_{135}-2 f_{1357}+18 f_{035}+3 f_{0357} \\
& +12 f_{046}-6 f_{146}-6 f_{025}-f_{0257}+18 f_{136}+12 f_{026}, \\
g_{0}^{0} * g_{1}^{2} * g_{1}^{4} * g_{0}^{0}= & -6 f_{146}+6 f_{046}+6 f_{147}-6 f_{047}+30 f_{03}+9 f_{035} \\
& -15 f_{036}+15 f_{037}+30 f_{14}-30 f_{04}+f_{0246}-f_{0247} \\
& -20 f_{13}-5 f_{024}-6 f_{135}+10 f_{136}-10 f_{137}, \\
g_{0}^{0} * g_{1}^{2} * \bar{g}_{1}^{4} * g_{0}^{0}= & 30 f_{03}+15 f_{035}-15 f_{036}+9 f_{037}+30 f_{14}-30 f_{04} \\
& -20 f_{13}-5 f_{024}-10 f_{135}+10 f_{136}-6 f_{137},
\end{aligned}
$$

$$
\begin{aligned}
g_{0}^{3} * g_{2}^{4} * g_{0}^{0}= & 3 f_{246}-3 f_{146}+3 f_{046}-f_{247}+f_{147}-f_{047}+20 f_{3} \\
& +4 f_{35}-10 f_{36}+4 f_{37}-10 f_{24}+10 f_{14}-10 f_{04}, \\
\left(f_{0}-78\right) * g_{2}^{4} * g_{0}^{0}= & 3 f_{0246}-f_{0247}+20 f_{03}+4 f_{035}-10 f_{036}+4 f_{037} \\
& -10 f_{024}-234 f_{246}+228 f_{146}-228 f_{046}+78 f_{247} \\
& -76 f_{147}+76 f_{047}-1560 f_{3}-312 f_{35}+780 f_{36} \\
& -312 f_{37}+780 f_{24}-760 f_{14}+760 f_{04}, \\
\left(f_{2}-78\right) * g_{1}^{4} * g_{0}^{0}= & -10 f_{13}+10 f_{03}-3 f_{135}+3 f_{035}+5 f_{136}-5 f_{036} \\
& -5 f_{137}+5 f_{037}+76 f_{246}-78 f_{146}+78 f_{046} \\
& -76 f_{247}+78 f_{147}-78 f_{047}+760 f_{3}+228 f_{35} \\
& -380 f_{36}+380 f_{37}-380 f_{24}+390 f_{14}-390 f_{04}, \\
\left(f_{2}-78\right) * \bar{g}_{1}^{4} * g_{0}^{0}= & -10 f_{13}+10 f_{03}-5 f_{135}+5 f_{035}+5 f_{136}-5 f_{036} \\
& -3 f_{137}+3 f_{037}+760 f_{3}+380 f_{35}-380 f_{36} \\
& +228 f_{37}-380 f_{24}+390 f_{14}-390 f_{04}, \\
\left(f_{2}-78\right) * g_{2}^{4} * g_{0}^{0}= & 20 f_{13}-20 f_{03}+4 f_{135}-4 f_{035}-10 f_{136}+10 f_{036}
\end{aligned}
$$$$
+4 f_{137}-4 f_{037}-228 f_{246}+234 f_{146}-234 f_{046}
$$$$
+76 f_{247}-78 f_{147}+78 f_{047}-1520 f_{3}-304 f_{35}
$$$$
+760 f_{36}-304 f_{37}+760 f_{24}-780 f_{14}+780 f_{04} \text {, }
$$$$
g_{0}^{0} * g_{1}^{7} * g_{0}^{0}=2 f_{13}-2 f_{14}+2 f_{15}-2 f_{16}+2 f_{17}+6 f_{02}-8 f_{03}
$$$$
+8 f_{04}-8 f_{05}+8 f_{06}-8 f_{07}-12 f_{1} \text {, }
$$$$
g_{0}^{0} *{\overline{g_{1}}}^{7} * g_{0}^{0}=8 f_{02}-8 f_{03}+8 f_{04}-8 f_{05}+8 f_{06}-6 f_{07}-16 f_{1} \text {, }
$$$$
{\overline{g_{1}}}^{8} * g_{0}^{0}=-18+9 f_{0}-9 f_{1}+9 f_{2}-9 f_{3}+9 f_{4}-9 f_{5}+9 f_{6}
$$$$
-7 f_{7},
$$$$
{\overline{g_{2}}}^{8} * g_{0}^{0}=72-36 f_{0}+36 f_{1}-36 f_{2}+36 f_{3}-36 f_{4}-36 f_{6}
$$$$
+30 f_{5}+3 f_{46}-3 f_{36}+3 f_{26}-3 f_{16}+3 f_{06}-f_{47}
$$$$
+f_{37}-f_{27}+f_{17}-f_{07}+22 f_{7} \text {, }
$$ 
[15]

$$
\begin{aligned}
{\overline{g_{3}}}^{8} * g_{0}^{0}= & -168+84 f_{0}-84 f_{1}+84 f_{2}+84 f_{4}+84 f_{6}+7 f_{27} \\
& -7 f_{17}+7 f_{07}-42 f_{7}-f_{047}+f_{147}-f_{247}+3 f_{046} \\
& -3 f_{146}+3 f_{246}-64 f_{3}-3 f_{37}-10 f_{24}+10 f_{14} \\
& -10 f_{04}+5 f_{47}+4 f_{25}-4 f_{15}+4 f_{05}-54 f_{5} \\
& -15 f_{46}+9 f_{36}-19 f_{26}+19 f_{16}-19 f_{06},
\end{aligned}
$$

[16]

$$
\begin{aligned}
g_{0}^{0} * g_{1}^{2} * g_{0}^{0} * g_{1}^{2} * g_{0}^{1} & =4 f_{1357}-6 f_{0357}+18 f_{047}+3 f_{0247}-18 f_{147}, \\
g_{0}^{1} * g_{1}^{2} * g_{1}^{2} * g_{0}^{1} & =-6 f_{157}+3 f_{0257}-f_{1357}-3 f_{0247}+9 f_{147},
\end{aligned}
$$$$
g_{0}^{4} * g_{1}^{2} * g_{0}^{1}=2 f_{57}-f_{057}+f_{157}-f_{257}+f_{357}-3 f_{47},
$$$$
{\overline{g_{1}}}^{4} * g_{1}^{2} * g_{0}^{1}=-3 f_{047}+3 f_{147}-3 f_{247}-10 f_{57}+5 f_{057}-5 f_{157}
$$$$
+5 f_{257}-3 f_{357}+15 f_{47} \text {, }
$$$$
g_{2}^{4} * g_{1}^{2} * g_{0}^{1}=3 f_{0257}-f_{0357}+20 f_{57}-10 f_{057}+4 f_{157}-10 f_{257}
$$$$
+4 f_{357}-30 f_{47}+12 f_{047}-3 f_{0247}+9 f_{247}-3 f_{147},
$$

$g_{0}^{2} * g_{1}^{4} * g_{0}^{1}=2 f_{37}-f_{037}+f_{137}-5 f_{27}$,

$g_{1}^{2} * g_{1}^{4} * g_{0}^{1}=-6 f_{37}+3 f_{037}-f_{137}+15 f_{27}-5 f_{027}$,

$g_{1}^{2} * \bar{g}_{1}^{4} * g_{0}^{1}=-f_{0247}+f_{0257}+3 f_{247}-3 f_{257}-f_{137}+3 f_{037}$

$-6 f_{37}+15 f_{27}-5 f_{027}$,

$g_{1}^{2} * g_{2}^{4} * g_{0}^{1}=-6 f_{357}+3 f_{0357}-f_{1357}+24 f_{37}-12 f_{037}$

$+4 f_{137}-30 f_{27}+9 f_{257}-3 f_{247}+10 f_{027}$

$-3 f_{0257}+f_{0247}$,

$g_{0}^{0} * g_{3}^{6} * g_{0}^{1}=2 f_{147}-8 f_{157}+2 f_{1357}-8 f_{137}+30 f_{17}-35 f_{07}$ $-5 f_{027}+f_{0257}-3 f_{0357}+10 f_{057}-4 f_{047}+13 f_{037}$,

$$
{\overline{g_{2}}}^{7} * g_{0}^{1}=7 f_{07}-7 f_{17}+7 f_{27}-7 f_{37}+f_{357}-f_{257}+f_{157}
$$

$$
\begin{gathered}
-f_{057}-4 f_{57}+4 f_{47}+14 f_{7}, \\
g_{1}^{2} * g_{1}^{2} * g_{0}^{0} * g_{1}^{2}=-54 f_{26}-27 f_{036}+54 f_{36}+18 f_{257}-9 f_{246}+6 f_{0357}
\end{gathered}
$$$$
-12 f_{357}+18 f_{026}+9 f_{136}-6 f_{0257}+3 f_{0246}
$$

$$
\begin{aligned}
g_{0}^{0} * g_{2}^{4} * g_{0}^{0} * g_{1}^{2}= & -60 f_{06}+30 f_{036}+60 f_{16}+20 f_{057}-16 f_{157} \\
& +4 f_{1357}+6 f_{146}-12 f_{046}-6 f_{0357}-12 f_{026} \\
& -18 f_{136}+2 f_{0257},
\end{aligned}
$$

$$
g_{0}^{1} * g_{1}^{2} * g_{0}^{1} * g_{1}^{2}=3 f_{0257}-6 f_{157}-3 f_{0246}-f_{1357}+9 f_{146} \text {, }
$$$$
{\overline{g_{1}}}^{4} * g_{0}^{1} * g_{1}^{2}=-3 f_{046}+3 f_{146}-3 f_{246}-3 f_{357}+5 f_{257}-5 f_{157}
$$$$
+5 f_{057}-10 f_{57}+15 f_{46} \text {, }
$$$$
g_{1}^{2} * g_{0}^{0} * g_{1}^{2} * g_{1}^{2}=18 f_{246}-54 f_{36}+27 f_{036}-18 f_{037}+36 f_{37}-18 f_{247}
$$$$
-3 f_{0357}+6 f_{357}-6 f_{0246}-9 f_{136}+6 f_{137}+6 f_{0247}
$$

$$
\begin{aligned}
g_{0}^{0} * g_{1}^{2} * g_{1}^{2} * g_{1}^{2}= & 18 f_{046}-18 f_{146}+18 f_{037}+3 f_{0357}+18 f_{147} \\
& -18 f_{047}+3 f_{0246}+18 f_{136}-12 f_{137}-3 f_{0247} \\
& -2 f_{1357}-27 f_{036},
\end{aligned}
$$

$$
g_{0}^{3} * g_{1}^{2} * g_{0}^{2}=f_{046}-f_{146}+f_{246}-3 f_{36},
$$

$$
\begin{aligned}
\left(f_{2}-78\right) * g_{0}^{2} * g_{1}^{2}= & 3 f_{036}-3 f_{136}+2 f_{137}-2 f_{037}+f_{1357}-f_{0357} \\
& -152 f_{37}-76 f_{357}+76 f_{247}-78 f_{147}+78 f_{047} \\
& +228 f_{36},
\end{aligned}
$$

$g_{0}^{1} * g_{2}^{4} * g_{0}^{2}=f_{0246}-4 f_{026}+10 f_{16}-3 f_{146}+f_{136}$,

$g_{0}^{1} * g_{2}^{4} * g_{1}^{2}=-3 f_{0246}+12 f_{026}+3 f_{0247}-f_{0257}+20 f_{17}+4 f_{137}$

$$
-10 f_{147}+4 f_{157}-10 f_{027}-30 f_{16}+9 f_{146}-3 f_{136},
$$




$$
\begin{aligned}
& g_{0}^{6} * g_{1}^{2}=2 f_{7}-f_{07}+f_{17}-f_{27}+f_{37}-f_{47}+f_{57}-3 f_{6}, \\
& {\overline{g_{1}}}^{6} * g_{1}^{2}=-14 f_{7}+7 f_{07}-7 f_{17}+7 f_{27}-7 f_{37}+7 f_{47}-3 f_{06} \\
& +3 f_{16}-3 f_{26}+3 f_{36}-3 f_{46}-5 f_{57}+21 f_{6}, \\
& g_{0}^{0} * g_{2}^{4} * g_{0}^{3}=2 f_{135}-8 f_{15}+10 f_{05}-3 f_{035}+f_{025}, \\
& g_{0}^{0} * g_{1}^{2} * g_{0}^{0} * g_{2}^{4}=-60 f_{04}+60 f_{14}+24 f_{035}-6 f_{0357}+18 f_{047}-18 f_{147} \\
& -6 f_{046}+6 f_{146}-10 f_{024}-16 f_{135}+4 f_{1357}+3 f_{0247} \\
& -f_{0246} \\
& \left(f_{2}-78\right) * g_{0}^{0} * g_{2}^{4}=-8 f_{135}+8 f_{035}+2 f_{1357}-2 f_{0357}-780 f_{04}+780 f_{14} \\
& -760 f_{24}+608 f_{35}-152 f_{357}+234 f_{047}-234 f_{147} \\
& +228 f_{247}-78 f_{046}+78 f_{146}-76 f_{246} \text {, } \\
& g_{0}^{1} * g_{1}^{2} * g_{2}^{4}=10 f_{024}-3 f_{0247}+f_{0246}+24 f_{15}+4 f_{135}-12 f_{025} \\
& -6 f_{157}-f_{1357}+3 f_{0257}-3 f_{146}+9 f_{147}-30 f_{14} \text {, } \\
& g_{0}^{4} * g_{2}^{4}=-8 f_{5}+4 f_{05}-4 f_{15}+4 f_{25}-4 f_{35}+2 f_{57}-f_{057} \\
& +f_{157}-f_{257}+f_{357}+f_{46}-3 f_{47}+10 f_{4}, \\
& \bar{g}^{4} * g_{0}^{4}=f_{04}-f_{14}+f_{24}-5 f_{4}, \\
& \left(f_{2}-78\right) * g_{0}^{5}=-f_{03}+f_{13}-76 f_{3} \text {, } \\
& g_{1}^{2} * g_{3}^{6}=-90 f_{3}+45 f_{03}+24 f_{35}-12 f_{035}-6 f_{357}+3 f_{0357} \\
& +24 f_{37}-12 f_{037}-6 f_{36}+3 f_{036}-15 f_{13}+4 f_{135} \\
& -f_{1357}+4 f_{137}-f_{136}+13 f_{025}-4 f_{026}+10 f_{027} \\
& -3 f_{0257}+f_{0247}-5 f_{024}-35 f_{02}-39 f_{25}+12 f_{26} \\
& -30 f_{27}+9 f_{257}-3 f_{247}+15 f_{24}+105 f_{2} \text {, } \\
& g_{0}^{1} * g_{1}^{7}=f_{02}-8 f_{1}, \\
& g_{0}^{1} * \bar{g}_{1}^{7}=f_{13}-f_{14}+f_{15}-f_{16}+f_{17}-f_{02}-6 f_{1} \text {, } \\
& g_{0}^{0} *{\overline{g_{1}}}^{8}=-f_{02}+f_{03}-f_{04}+f_{05}-f_{06}+f_{07}+2 f_{1}-9 f_{0} \text {, } \\
& g_{0}^{0} * g_{2}^{8}=-16 f_{1}+2 f_{13}+f_{02}-3 f_{03}+36 f_{0} \text {, } \\
& g_{0}^{0} * \bar{g}^{8}=8 f_{02}-8 f_{03}+8 f_{04}-8 f_{05}-16 f_{1}+2 f_{17}+f_{057} \\
& -f_{047}+f_{037}-f_{027}-7 f_{07}+5 f_{06}+36 f_{0} \text {, } \\
& g_{0}^{0} * g_{4}^{8}=-112 f_{1}+30 f_{13}-8 f_{135}-10 f_{14}+2 f_{147}-8 f_{157} \\
& +2 f_{1357}-8 f_{137}+30 f_{17}+2 f_{136}-10 f_{16} \\
& +34 f_{15}+21 f_{04}-45 f_{05}+15 f_{06}-3 f_{036}+f_{026} \\
& -35 f_{07}-5 f_{027}+f_{0257}-3 f_{0357}+10 f_{057}-4 f_{047} \\
& +13 f_{037}-51 f_{03}+12 f_{035}-4 f_{025}+21 f_{02}+126 f_{0} \text {, } \\
& g_{1}^{9}=-10+f_{0} \text {. }
\end{aligned}
$$

The proof was obtained as follows. FLAGTOOL created 227 linear inequalities which contain the 53 inequalities above. The infeasibility of the entire list of inequalities and the creation of the smaller list of 53 inequalities which are already infeasible was first carried using phase I of the LP-solver CPLEX. Since it was (at least theoretically) possible that the infeasibility found by CPLEX is an artifact of numerical problems we proved the infeasibility using the symbolic mathematical program MAPLE V.

Note that we need the nonnegativity of the forms $g_{3}^{d}$ and it turns out that using only the nonnegativity of $g_{i}^{d}, i \leq 2$, is not sufficient to prove infeasibility. Thus, Theorem 1 is still unproved for general (nonrational) polytopes.

It seems impossible to prove the infeasibility "by hand." The details of this proof (which for a computer generated proof is quite short) do not seem to contribute much to our (human) understanding why the theorem is true. 
Proof of Theorem 2. We added the inequalities $f_{0}^{3}-5 \geq 0$ and $f_{0}^{3}-5 \geq 3$ (for all intervals of rank 4) and all inequalities generated by convolutions. The theorem follows from the infeasibility of a system of 50 linear inequalities which is contained in the 243 inequalities produced by FLAGTOOL. This time we use only the nonnegativity of $g_{i}^{d}$ for $i \leq 2$. The infeasibility was again proved by using MAPLE V. We skip the details.

Remark. It is possible that the statements of Theorems 1 and 2 remain true for arbitrary Eulerian lattices [2], [17] for some sufficiently large dimension. The same comment applies to all the conjectures mentioned in Section 1. For Eulerian lattices it is no longer true that the $g_{i}^{d}$ are nonnegative for $i \geq 2$ but the nonnegativity of the $g_{1}^{d}$ may suffice.

Proof of Theorem 3. We added the inequalities $f_{0}^{3}-9 \geq 0$ and $f_{0}^{3}-9 \geq 3$ to the other inequalities for 5-polytopes and used convolution to obtain a system of 14 inequalities which turned out to be infeasible. This time, we used only the nonnegativity of $g_{1}^{d}$ and hence the proof applies to arbitrary Eulerian lattices.

\section{References}

1. D. Barnette, A proof of the lower bound conjecture for convex polytopes, Pacific J. Math. 46 (1971), 349-354.

2. M. M. Bayer and L. J. Billera, Generalized Dehn-Sommerville relation for polytopes, spheres, and Eulerian partially ordered sets, Invent. Math. 79 (1985), 143-157.

3. A. Bezdek, K. Bezdek, E. Makai, and P. McMullen, Facets with fewest vertices, Monatsh. Math. 109 (1990), 89-96.

4. R. Descartes, De Solidorum Elementis, in Oeuvres de Descartes, C. Adam and P. Tannery, Paris, 18971913, Vol. 10, pp. 265-276.

5. B. Grünbaum, Convex Polytopes, Interscience, London, 1967.

6. B. Grünbaum, Polytopes, graphs, and complexes, Bull. Amer. Math. Soc. 97 (1970), 1131-1201.

7. B. Grünbaum, Arrangements and Spreads, American Mathematical Society, Providence, RI, 1972.

8. B. Grünbaum and G. C. Shephard, Lectures on lost mathematics, Manuscript, University of Washington, 1978.

9. G. Kalai, Rigidity and the lower bound theorem, I, Invent. Math. 88 (1987), 125-151.

10. G. Kalai, A new basis of polytopes, J. Combin. Theory Ser. A 49 (1988), 191-209.

11. G. Kalai, On low-dimensional faces that high-dimensional polytopes must have, Combinatorica 10 (1990), 271-280.

12. G. Kalai, P. Kleinschmidt, and G. Meisinger, Flag numbers and FLAGTOOL, in: PolytopesCombinatorics and Computation (G. Kalai and G. Ziegler, eds.), DMV-Seminars Series, BirkhäuserVerlag, Basel, 2000, in press.

13. P. McMullen, The number of faces of simplicial polytopes, Israel J. Math. 9 (1971), 559-570.

14. G. Meisinger, Flag Numbers and Quotients of Convex Polytopes, Schuch-Verlag, Weiden, 1994.

15. R. Stanley, Generalized $h$-vectors, intersection cohomology of toric varieties, and related results, in Commutative Algebra and Combinatorics (M. Nagata and H. Matsumura, eds.), Advanced Studies in Pure Mathematics 11, Kinokuniya, Tokyo, and North-Holland, Amsterdam, 1987, pp. 187-213.

16. R. Stanley, Combinatorics and Commutative Algebra, second edition, Birkhauser, Boston, 1994.

17. R. Stanley, A survey of Eulerian posets, in Polytopes, Abstract Convex and Computational (T. Bisztriczky et al., eds.), Kluwer, Dordrecht, 1994, pp. 301-333. 